\title{
Generation and trajectory control of water drops able to bounce on a flat water surface
}

\author{
Clément Gouiller* , Axel Guittonneau, and Léa Jacquot \\ Univ. Lyon, ENS de Lyon, Univ. Claude Bernard, CNRS, Laboratoire de Physique, Département de Physique, \\ 69342 Lyon, France
}

Received: 19 December 2016 / Accepted: 6 July 2017

\begin{abstract}
Under some conditions, a water droplet can bounce on a flat water surface. This paper contains a qualitative analysis of the bouncing phenomenon that leads to try to obtain drops with a nearly tangential incidence, the smallest radius and the highest velocity possible. We describe the experimental setup that we built, able to throw unique droplets at typical speed $v \sim 1 \mathrm{~m} . \mathrm{s}^{-1}$, radius $R \sim 4 \times 10^{-4} \mathrm{~m}$ and impact angle $\theta \sim 15^{\circ}$ with respect to the surface. Up to 4 bounces were experimentally observed. The experimental results' plot shows the statistical behaviour of the bouncing process: the initial conditions are not sufficient to predict the trajectory and consequently the number of bounces.
\end{abstract}

Keywords: bouncing water droplets / Leidenfrost slide / Weber number / superhydrophobic surface

\section{Introduction}

When a water droplet impacts a flat water surface, four different behaviours can be observed [1]: coalescence, floating, bouncing and splashing (Fig. 1). An analogy can be made with a skipping stone: under specific experimental conditions (including angle, shape, linear and angular momentum), the stone can bounce one or several times on the water surface [2]. However, under other experimental conditions, the stone can break the water surface and sink. In the case of a water drop impacting a water surface, the droplet can merge with the rest of the water after the collision: this is coalescence. This impact can also cause a splashing phenomenon, with the production of secondary droplets [1]. However, a droplet can also bounce on the water because of the presence of a thin air layer that prevents the contact with the water surface. The purpose of this paper is to devise an experimental setup to make a water droplet bounce several times on a water surface. To achieve this goal, we built an apparatus capable of throwing water drops on a still water surface at a controlled speed, size and impact angle. We had to determine a range of impact parameters with a high probability to bounce at least once. Reading Jayaratne and Mason [3] led us to consider three impact parameters: the radius $R$ of the droplet, its velocity $v$ and its impact angle $\theta$ between the trajectory of the drop and the tangent to the surface. A qualitative analysis led us to consider small

* e-mail: clement.gouiller@ens-lyon.fr drops with a small impact angle and a high speed: we built a setup able to throw unique droplets at typical speed $v \sim 1 \mathrm{~m} . \mathrm{s}^{-1}$, radius $R \sim 4 \times 10^{-4} \mathrm{~m}$ and impact angle $\theta \sim 15^{\circ}$. Up to 4 bounces were experimentally observed. We also tried to investigate whether there are boundaries in the parameter space between different number of bounce zones. We looked at 50 droplets to try to find the zone that gives the maximal number of bounces. Our results do not show a clear separation between bounce and no-bounce zones in the parameter space. This is coherent with the work of Zhbankova and Kolpakov [4]: following the pioneering work of Jayaratne and Mason [3], they investigated statistically the bouncing behavior. In contradiction with the precedent work [3], their results suggest that there is no clear boundary between bounce and no-bounce zones in the parameter space. A more accurate description would be statistical: to a given set of parameters is associated a probability to bounce. However, our results show trends: the number of bounces increases when the impact angle or the Weber number We (a dimensionless parameter, defined as the ratio between inertia of the drop and surface tension forces) decreases.

\section{Methods}

\subsection{Physics of the bounce}

Before starting the experiments, it is important to understand the phenomena occurring when a droplet impacts the surface, in order to assess the range of impact parameters needed and devise the setup adequately. 


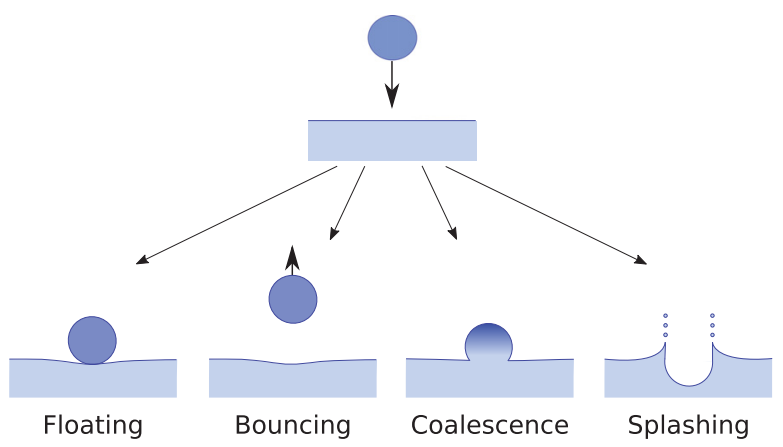

Fig. 1. Impact of a drop on a liquid surface, inspired from Rein [1].

The main idea is to take into account the air layer between the drop and the surface. This one can be considered in the drop's frame as a constant air flow in the opposite direction that needs to support the drop. The air layer is stressed between the drop and the surface, inducing an overpressure below the drop, which increases with the velocity of the drop. Coalescence happens only when this air layer breaks.

To avoid such a rupture, the weight of the droplet applied on it needs to be reduced. Thus, we chose droplets with a small radius, typically $R \sim 4 \times 10^{-4} \mathrm{~m}$. We also wanted droplets able to bounce several times on the water surface. At each bounce, part of the droplet's kinetic energy is lost, used to deform the surface and then dissipated by viscosity. Thereby, we had to consider droplets with a large speed (typically $v \sim 1.2 \mathrm{~m} . \mathrm{s}^{-1}$ ), but small radius as discussed previously. Finally, the greater the tangential speed the greater the induced overpressure below the droplet. Consequently, having nearly-tangential impact angles will favour bouncing, typically $\theta \sim 15^{\circ}$.

\subsection{Experimental setup}

We built an experimental setup in order to obtain droplets of small radius, high velocity and small impact angle with respect to the water surface. Our setup can be divided into three parts (Fig. 2).

The first part (1) is a programmable syringe pump linked to a needle. The syringe pump forces a constant flow of water through the needle. This generates droplets at a rate proportional to the water flow, without changing their mass - as long as the flow is small enough to consider their formation as a quasi-static phenomenon. Water accumulates on the tip of the needle, until its weight becomes higher than surface tension forces. We did not manage to make drops small enough to bounce until we added a DC tension of $5 \mathrm{kV}$ between the tip of the needle and a metal ring (2) placed $1 \mathrm{~cm}$ below the needle. Then, droplets are polarized in the needle, and pulled by the electrostatic field. This phenomenon allows to generate smaller and faster droplets (see Section 3). We then converted the droplets' vertical velocity into horizontal velocity, in order to obtain small impact angles. Inspired from Harris et al. [5], we chose to use a slide (Fig. 2, part (3)): the drop arrives vertically on the top part of the slide, rides on it and leaves it with a horizontal velocity. The main issue here is to avoid

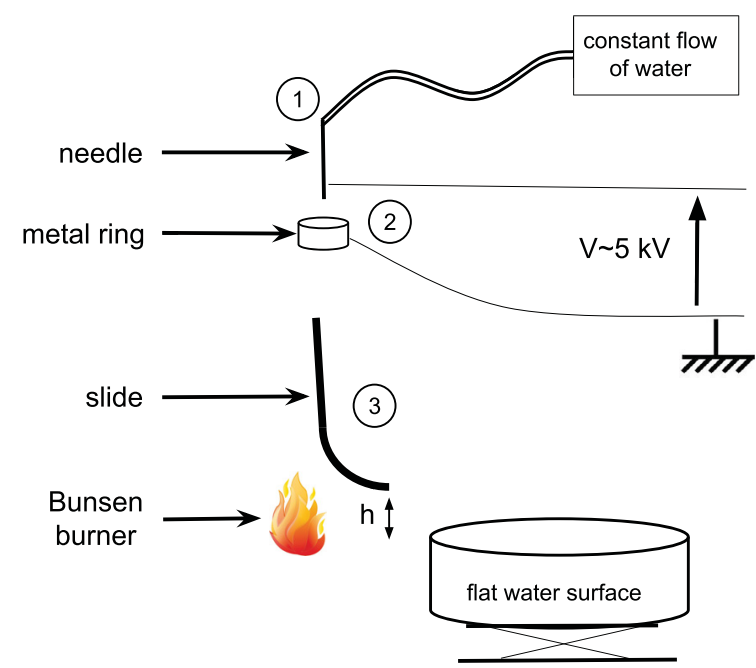

Fig. 2. Diagram of the experimental setup. The numbers $(1,2,3)$ correspond to the different parts of the setup. Part 1 generates drops, part 2 triggers the mean radius of drops and accelerates them. Part 3 allows to select the impact angle of the drops.

speed depletion, so we used the Leidenfrost effect [6]: a stainless steel slide is heated with a Bunsen burner at a temperature ranging from 200 to $400{ }^{\circ} \mathrm{C}$. At those temperatures, a thin vapour layer forms between the drop and the slide, avoiding direct contact and as a consequence reducing speed depletion on the slide. The impact angle $\theta$ of the drop can be selected by modifying the height $h$ between the bottom of the slide and the water surface. The higher the tip of the slide the greater the impact angle (see Fig. 3).

\subsection{Measures}

The dynamics of each drop has been studied using records made by a high speed camera (FLARE 2M360 MCL) oriented perpendicularly to the trajectory of the drop. By restricting the field of view to a window of $758 \times 158 \mathrm{px}$ (see Fig. 3a), we were able to obtain images at $300 \mathrm{fps}$. Close attention needs to be payed to the lighting, in order for the drop to appear white on a black background. We obtained for each drop its speed and impact angle just before reaching the surface. Because of motion blur (due to the $1 / 300$ s exposure time for each frame), the drop did not appear spherical, but as an oblong form. The radius of each drop was hence measured by considering the width of the oblong form (we considered that the drop stays spherical). For each drop, we counted the number of bounces observed.

\section{Results}

\subsection{Drop generation}

We studied the influence of two different parameters on the mean size of the drops created: the radius of the needle $r_{\text {needle }}$ and the voltage between the needle and the metal ring $\Delta V$. In theory, the drop should leave the needle as soon as surface tension forces of the needle/water interface become smaller than the weight of the drop plus the electrostatic force (see Fig. 4). 


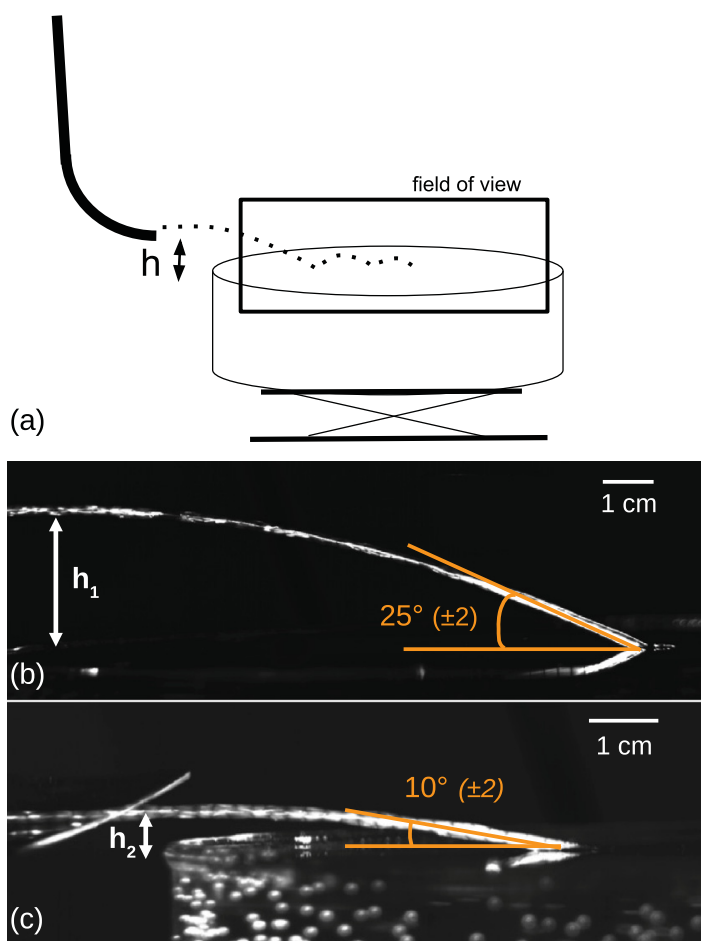

Fig. 3. (a) Diagram showing the field of view corresponding to the following images. (b, c) Trajectory of drops for two different heights of the slide: $h_{1}=2.6 \mathrm{~cm}$ and $h_{2}=0.7 \mathrm{~cm}$. We observed a smaller impact angle for $h_{2}\left(10 \pm 2^{\circ}\right)$ than for $h_{1}\left(25 \pm 2^{\circ}\right)$. Both images are obtained from successive frames ( $300 \mathrm{fps})$ layered in a single one to reconstruct the full trajectory.

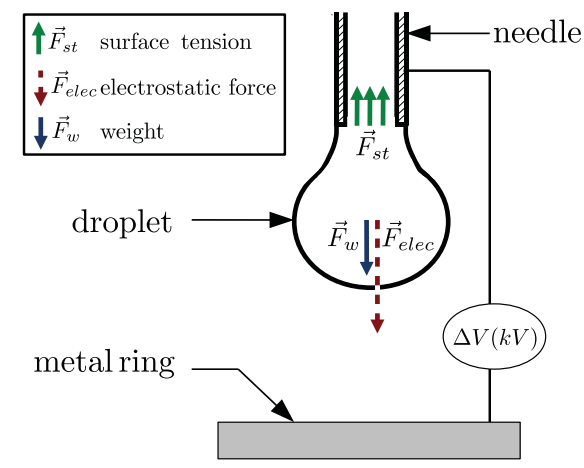

Fig. 4. Diagram of the forces applied to the water droplet before falling from the needle.

One can write that the drop falls as soon as:

$$
\begin{gathered}
F_{s t}=F_{w}+F_{\text {elec }} \\
\Leftrightarrow m_{\text {drop }}=\frac{F_{\text {st }}-F_{\text {elec }}}{g}
\end{gathered}
$$

with:

- $F_{s t}$ the attractive force between the drop and the needle, which can be written as $2 \pi r_{\text {needle }} \gamma$ with $r_{\text {needle }}$ the radius of the needle and $\gamma$ the surface tension of the water/needle interface. This force does not depend on the size of the drop.

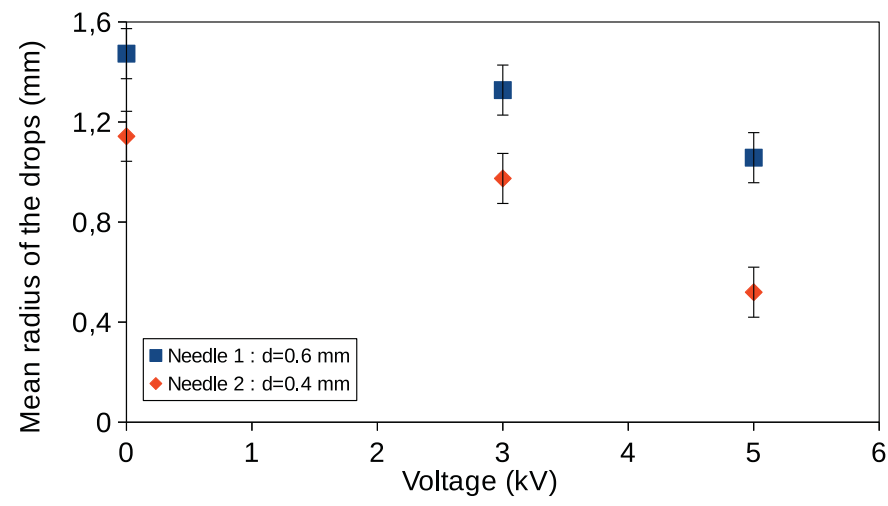

Fig. 5. Influence of the voltage $\Delta V$ and radius $d / 2$ of the needle on the mean radius of generated drops. This mean radius is experimentally determined by collecting 50 drops and measuring their total mass.

- $F_{w}$ the weight of the drop which is equal to its mass $m_{d r o p}$ multiplied by the gravitational acceleration constant $g$.

- $F_{\text {elec }}$ the attractive force between the drop and the metal ring. It increases with the imposed voltage between them.

We see in equation (2) that the size of the drops depends only on the imposed voltage and decreases when the voltage increases. Equation (2) is known as Tate's law [7] when $F_{\text {elec }}=0$. For different needle radii and voltages, we collected 50 drops and measured their mass. This gave us the mean mass of each drop, plotted in Figure 5. We observed that the mean radius of the drops decreases with thinner needles and greater electrostatic forces, validating qualitatively equation (2).

\subsection{Typical sizes and speeds of the problem}

To avoid any side effect, the flat surface was chosen to have high depth $(10 \mathrm{~cm})$ and length $(60 \mathrm{~cm})$ with respect to the waves created by drops while coming close to the surface. Experimentally, the drops created had a typical radius of $R=(4 \pm 1) \times 10^{-4} \mathrm{~m}$, velocity of $v=1.2 \pm 0.2 \mathrm{~m} . \mathrm{s}^{-1}$ and impact angle of $\theta=15 \pm 10^{\circ}$.

\subsection{Impact angle control}

A simple reasoning shows that a greater impact angle is obtained when increasing the height of the plate (see Fig. 3). It is important to notice that the impact angle also depends on the speed of the drop at the end of the slide, which explains that we experimentally observed different impact angles for the same height of the slide.

\subsection{Bounces}

With the setup described previously, we managed to obtain up to 4 bounces. Examples of trajectories are shown in Figure 6 . They were obtained by superposing successive frames to reconstruct the full trajectory.

To summarize our results on a single meaningful scatter plot, we used the Weber number $W e$. We is a dimensionless parameter defined as the ratio between inertia of the drop and surface tension forces: $W e=\frac{\rho v^{2} R}{\gamma}$, with $\rho$ the volumetric mass of water, $v$ the normal velocity of the 


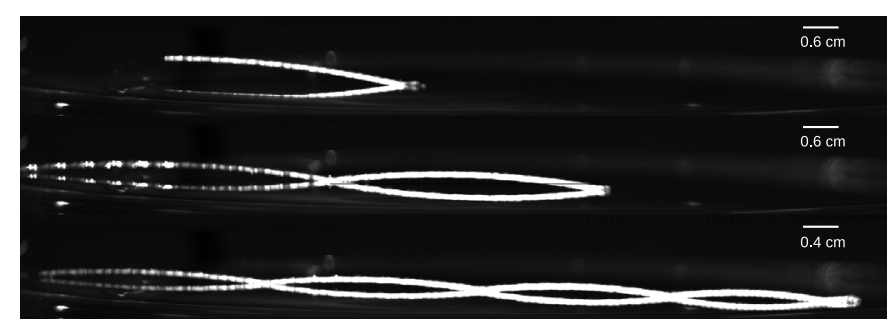

Fig. 6. Trajectory of water drops arriving on a flat water surface. The drop coalesces (top), bounces two (middle) or four times (bottom). Pictures were obtained by superposition of successive frames. The drop comes from the left and its reflection is seen in the water surface.

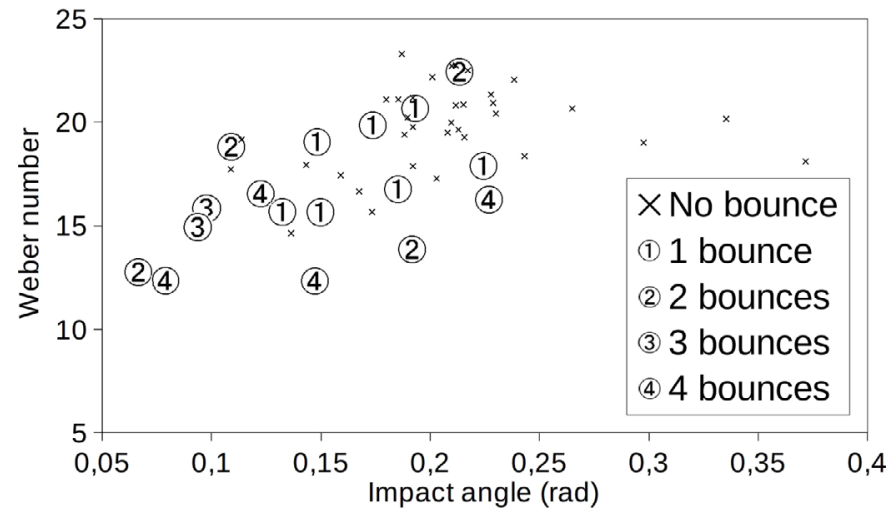

Fig. 7. Weber number against impact angle for a sample of drops. The velocity and size of each drop were determined thanks to the records obtained from a FLARE 2M360 MCL high frame camera and numerical measurements. Uncertainties of $20 \%$ on the Weber number and $\pm 0.02 \mathrm{rad}$ on the impact angles have to be considered.

drop with respect to the surface and $\gamma$ the surface tension of water. The Weber number is an appropriate parameter because coalescence happens when the air layer between the drop and the water surface breaks. Inertia is the cause of this rupture and surface tension forces help to avoid it.

We summarized our results on a scatter plot of the number of bounces in the $(\theta, W e)$ plane (Fig. 7). As in [4], we did not observe clear boundaries between bouncing or no-bouncing areas.

\section{Discussions}

The number of bounces obtained (up to 4) suggests that the chosen range of radius, velocity and impact angle was appropriate. The general ideas of our experimental setup allowed to study various sets of parameters $(R, v, \theta)$. In Figure 7, we did not observe boundaries between the different number of bounces. Due to uncertainties on the Weber number (up to 20\%), we cannot be sure that they do not exist, but it would be consistent with the work of Zhbankova and Kolpakov [4] who studied whether a droplet bounces once or not. In addition to their conclusions, we are able to say that each bounce has a statistical description, that is why the statistical behaviour can be extended to the number of bounces for a given set of parameters.

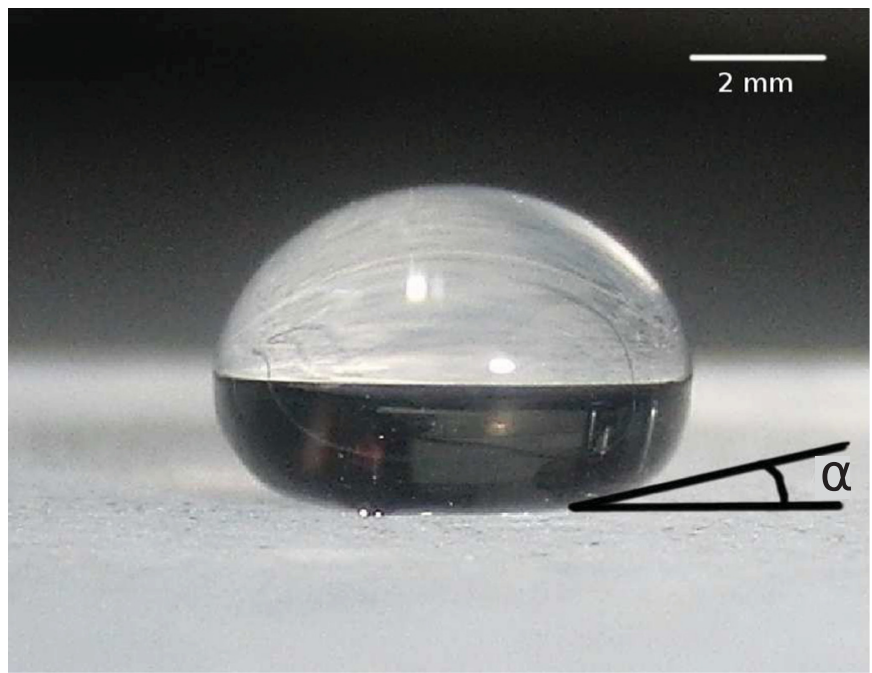

Fig. 8. Drop laying on a superhydrophobic surface (candle soot and shoe waterproofing product on a PMMA plate). The contact angle $\left(180^{\circ}-\alpha\right)$ is approximately $160^{\circ}$.

Moreover, some trends are visible in Figure 7 . In this region of the parameter space, the number of bounces increases when the impact angle or the Weber number decreases. It is consistent with our definition of the Weber number: for high Weber numbers, surface tension forces are not strong enough with respect to the inertia of the drop to avoid coalescence. However, no direct comparison of results can be made with [3] or [4], since our experiments explored a different part of the parameter space.

\subsection{Improving the setup}

Some ideas can allow to improve the experimental setup of this study.

The main issue preventing to draw a conclusion is the uncertainties on the impact parameters. For example, our setup was not able to generate drops of a precise radius. It was just possible to make the mean radius increase or decrease by changing the voltage and the size of the needle. Another source of variability is the evaporation of part of the drop on the heated slide. As a consequence, the radius needed to be measured experimentally for each drop, by looking on the records. A drop is visible on the video on a small number of pixels, inducing an uncertainty on the 
radius. Using two cameras - one zoomed to measure the drops radius when leaving the slide and another to measure the number of bounces - would decrease this uncertainty.

One should also consider using a V-shaped slide to allow a more precise control of the drops' trajectories when leaving the slide.

Another issue is the lack of data available to draw statistical conclusions. The analysis of the trajectories should be automated by an image analysis program to allow the study of more drops.

\section{Dead end}

Before developing the experimental device from which all the above results have been obtained, we investigated two different setups.

\subsection{Hydrophobic slides}

We tried to build superhydrophobic slides instead of using the Leidenfrost effect, to see if we could obtain better results. Different surfaces have been studied with commercial and home-made products on PMMA surfaces. We compared their hydrophobic power by measuring the contact angle of a drop with each surface. We obtained the best result with a PMMA plate on which we put candle soot and a shoe waterproofing product. The contact angle was approximately $160^{\circ}$ (see Fig. 8). We tried to build a curved slide using this hydrophobic power and to use it with our experimental setup, but it was less efficient than the "Leidenfrost slide". The drop lost more speed on the slide because friction was higher than with a "Leidenfrost slide".

\subsection{Silicon oil drops and surface}

Inspired by the work of Harris et al. [5], we designed a setup to obtain bounces with silicon oil droplets on a silicon oil surface. We obtained bounces with bigger and slower drops than for water. The slide used was a PMMA slide covered with a highly-viscous silicon oil, and we did not use any electrostatic device, just a programmable syringe pump linked to a needle. The purpose of this study was to answer the question: "Will the results change qualitatively if using honey instead of water". A more complete study could be carried as a lab project or internship to follow this idea.

\section{Conclusion}

We proposed in this paper an experimental setup that allowed us to make a water droplet bounce up to 4 times on a flat water surface. The understanding of the setup involves diversified fields of physics such as fluid mechanics and electrostatics. However, there is still room for improvement: our setup could probably be automated to gather more data, and improved to reduce uncertainties. Indeed, our data did not show any evidence of a clear frontier between bouncing and no-bouncing zones on a $(\theta, W e)$ diagram. This could be due to the statistical description of the bounce or to measurement uncertainties. The first explanation would be consistent with [4]: to a given set of parameters is associated a probability to bounce. Our measurements gave some trends: in the region of the control parameters space studied, the number of bounces increases when the impact angle and the Weber number decrease.

We would like to thank A. Bourges, A. Chemin, C. Malciu and J. Sautel, our amazing teammates for the International Physicists' Tournament 2016 for their advices and remarks while working on this project. We would also like to thank E. C. Jaupart and S. Liot for their help and of course our team leaders N. Plihon, N. Taberlet and A. Caussarieu who have always been there for us.

\section{References}

1. M. Rein, Phenomena of liquid drop impact on solid and liquid surfaces, Fluid Dyn. Res. 12, 61 (1993)

2. H.R. Crane, What can a dimple do for a skipping stone? Phys. Teacher 26, 300 (1988)

3. O.W. Jayaratne, B.J. Mason, The coalescence and bouncing of water drops at an air/water interface, Proc. R. Soc. Lond. A: Math. Phys. Eng. Sci. 280, 545 (1964)

4. S.L. Zhbankova, A.V. Kolpakov, Collision of water drops with a plane water surface, Fluid Dyn. 25, 470 (1990)

5. D.M. Harris, L. Tanya, J.W.M. Bush, A low-cost, precise piezoelectric droplet-on-demand generator, Exp. Fluids 56, 83 (2015)

6. A. Hashmi et al., Leidenfrost levitation: beyond droplets, Sci. Rep. 2, 797 (2012)

7. T. Tate, On the magnitude of a drop of liquid formed under different circumstances. Lond. Edinb. Dublin Philos. Mag. J. Sci. 27, $176(1864)$

Cite this article as: Clément Gouiller, Axel Guittonneau, Léa Jacquot, Generation and trajectory control of water drops able to bounce on a flat water surface, Emergent Scientist 1, 1 (2017) 